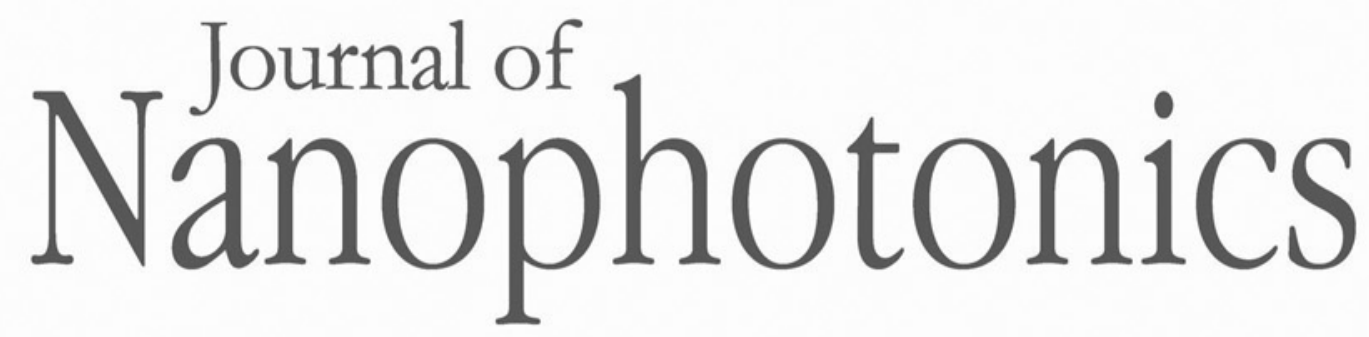

Nanophotonics.SPIEDigitalLibrary.org

\title{
Multiresonant layered plasmonic films
}

\author{
Brent M. DeVetter \\ Bruce E. Bernacki \\ Wendy D. Bennett \\ Alan Schemer-Kohrn \\ Kyle J. Alvine
}




\title{
Multiresonant layered plasmonic films
}

\author{
Brent M. DeVetter, Bruce E. Bernacki, Wendy D. Bennett, \\ Alan Schemer-Kohrn, and Kyle J. Alvine* \\ Pacific Northwest National Laboratory, Richland, Washington, United States
}

\begin{abstract}
Multiresonant nanoplasmonic films have numerous applications in areas such as nonlinear optics and sensing and as spectral tags. Although techniques such as focused ion beam milling and electron beam lithography can produce high-quality multiresonant films, these techniques are expensive, serial processes that are difficult to scale at the manufacturing level. Here, we present the fabrication of multiresonant nanoplasmonic films using a layered stacking technique. Periodically spaced gold nanocup substrates were fabricated using selfassembled polystyrene nanospheres followed by oxygen plasma etching and metal deposition via magnetron sputter coating. By adjusting etch parameters and initial nanosphere size, it was possible to achieve an optical response ranging from the visible to the near-infrared. Singly resonant, flexible films were first made by performing peel-off using an adhesive-coated polyolefin film. Through stacking layers of the nanofilm, we demonstrate fabrication of multiresonant films at a fraction of the cost and effort compared with top-down lithographic techniques. (C) The Authors. Published by SPIE under a Creative Commons Attribution 3.0 Unported License. Distribution or reproduction of this work in whole or in part requires full attribution of the original publication, including its DOI. [DOI: 10.1117/1.JNP.11.016015]
\end{abstract}

Keywords: plasmonics; nano-optics; gold nanocups; nanomaterials.

Paper 16181 received Nov. 23, 2016; accepted for publication Feb. 22, 2017; published online Mar. 14, 2017.

\section{Introduction}

Plasmonics has emerged as a promising field with a diverse set of applications, including colorimetric sensors, ${ }^{1}$ data storage, ${ }^{2,3}$ photovoltaics, ${ }^{4}$ and surface-sensitive chemical identification. ${ }^{5}$ Nanostructures consisting of metals such as gold, silver, and aluminum exhibit a resonance effect called the localized surface plasmon resonance (LSPR) in which conduction band electrons collectively oscillate at certain frequencies and vary based on the metal's complex index of refraction and geometry. Plasmonic nanostructures can have brilliant optical properties as a consequence of the LSPR and were conventionally tailored to visible wavelengths, but more recently, these have been engineered to range from the ultraviolet to the infrared (IR). ${ }^{6}$ Modifying the nearby dielectric environment, shape, size, or composition of a plasmonic nanostructure alters its LSPR, facilitating tunability of the resonances across a wide frequency spectrum with minimal effort. Gold nanostructures, in particular, have been heavily used in this field due to their excellent chemical stability, well-established processing techniques, and excellent visible/near-IR response.

Bottom-up synthesis techniques are robust and scalable, but the fabrication of solutionprocessed and periodic self-assembled large area $\left(>\right.$ several $\left.\mathrm{cm}^{2}\right)$ plasmonic substrates remains challenging. ${ }^{8-11}$ For research purposes, top-down approaches are desirable, but they present their own unique challenges. To fabricate arrays of high-quality periodic nanostructures, researchers often turn to electron beam lithography (EBL) or focused ion beam (FIB) milling, where precisely defined nanostructures can be patterned on substrates. ${ }^{12,13}$ Both EBL and FIB milling, however, are serial processes that are challenging and expensive to scale up to manufacturing levels.

To overcome the limitations of EBL and FIB milling, researchers often use a technique called colloidal lithography, which is a hybrid technique that uses colloidal self-assembly to produce relatively large periodic arrays of nanostructures at a low cost with minimal equipment. ${ }^{14}$

*Address all correspondence to: Kyle J. Alvine, E-mail: kyle.alvine@pnnl.gov 
Commercially available polymeric micro/nanospheres with a high degree of uniformity and monodispersity readily self-assemble into two-dimensional monolayers or three-dimensional (3-D) colloidal crystals when using techniques such as spin coating, controlled solvent evaporation, or dipping (Langmuir-Blodgett). Halas and coworkers, ${ }^{15-17}$ in particular, first pioneered the development of gold nanocups using randomly dispersed (i.e., nonperiodic) polystyrene nanospheres on substrates after depositing a thin layer of gold and demonstrated their unique light-bending optical properties arising from their asymmetry.

Gold nanocups have been heavily studied because of these unique optical properties. In particular, nanocups behave as 3-D split ring resonators ${ }^{18}$ that, with proper illumination and orientation angles, can exhibit both electric and magnetic dipole plasmon modes. Researchers have shown that by harnessing either the electric or the magnetic dipole modes, it is possible to engineer desired optical properties such as directing far-field radiation of light. ${ }^{16}$ These properties can be further engineered by tuning the local dielectric environment and substrate on which the nanocup is bound. ${ }^{15}$

In this work, we use colloidal lithography and magnetron sputter coating to fabricate periodic arrays of gold nanocups. The gold nanocup arrays were then transferred to a flexible, optically transparent film with a pressure-sensitive adhesive and a peel-off technique. The peel-off technique used is advantageous because it is rapid (can be performed in a few minutes) and uses commonly available materials, such as adhesive tape. Furthermore, this technique allows us to independently design singly resonant films with precisely tailored properties that combine when stacked to form spectrally complex films. In contrast, other techniques to produce single layers of multiresonant plasmonic films require additional fabrication steps such as replica molding to fabricate a patterned surface or sequential deposition of nanospheres, ${ }^{19,20}$ adding to the overall cost and time of fabrication. We conclude with the characterization and discussion of multiresonant plasmonic films for applications requiring unique spectral signatures.

\section{Methods}

Periodic arrays of gold nanocups were fabricated according to the schematic outlined in Fig. 1. Briefly, silicon (100) wafers (EL-CAT, Inc.) were cleaned with an oxygen plasma (Nordson MARCH AP-600, $250 \mathrm{~W}$ and $30 \mathrm{sccm} \mathrm{O}_{2}$ ) for $15 \mathrm{~min}$ to ensure a hydrophilic surface. Commercially purchased (Bangs Laboratories, Inc.) polystyrene nanospheres (10 wt. \% solids) with diameters ranging from 170 to $580 \mathrm{~nm}$ were diluted to 5 to $7 \mathrm{wt}$. \% solids with doubledistilled $\mathrm{H}_{2} \mathrm{O}$ before use. After briefly vortexing and sonicating the solution to minimize nanosphere agglomeration, $\sim 0.5 \mathrm{~mL}$ of nanosphere solution was deposited onto a cleaned silicon wafer and spin-casted to form self-assembled hexagonally close-packed monolayers, as shown in Fig. 1. Typical spin speeds varied as a function of size and concentration of the nanospheres. We found that for a spin time of $1 \mathrm{~min}$, smaller nanospheres (170 $\mathrm{nm}$ diameter) produce a better quality film when spun at high rpm (up to $8000 \mathrm{rpm}$ ) and larger nanospheres $(580 \mathrm{~nm}$ diameter) typically spin better at lower speed $(<3000 \mathrm{rpm})$. The quality of the film was evaluated using optical microscopy before proceeding with etching and adjustments to the spin speed were made, if necessary. Some grain boundaries and point defects (vacancies) were observed to be unavoidable, but the overall microscopic film quality remained high in most regions. Although the quality of the self-assembled film is nanosphere size-dependent, we observed areas with no or exceedingly minimal defects up to a few microns in dimension. The spin-casted substrates were annealed at $107^{\circ} \mathrm{C}$ in an oven for 2 min to improve neighbor-to-neighbor and substrate adhesion. We found annealing to be a crucial step for achieving optimal film quality. Once cooled to room temperature, the samples were etched for roughly 3 to 5 min with an oxygen plasma (typically $75 \mathrm{~W}, 20 \mathrm{sccm} \mathrm{O}_{2}$ ) until the desired size was achieved. Gold deposition (20 nm thick) was performed at normal incidence with respect to the substrate using magnetron sputter coating. Representative scanning electron micrographs (JEOL JSM$7600 \mathrm{~F}, 2 \mathrm{kV}$ accelerating voltage) of each step are shown in Fig. 1.

\section{Results and Discussion}

Scanning electron micrographs and the corresponding reflectance spectra on the sacrificial silicon substrate after metallization are shown in Fig. 2. The plasmon resonance of interest for 
(a)
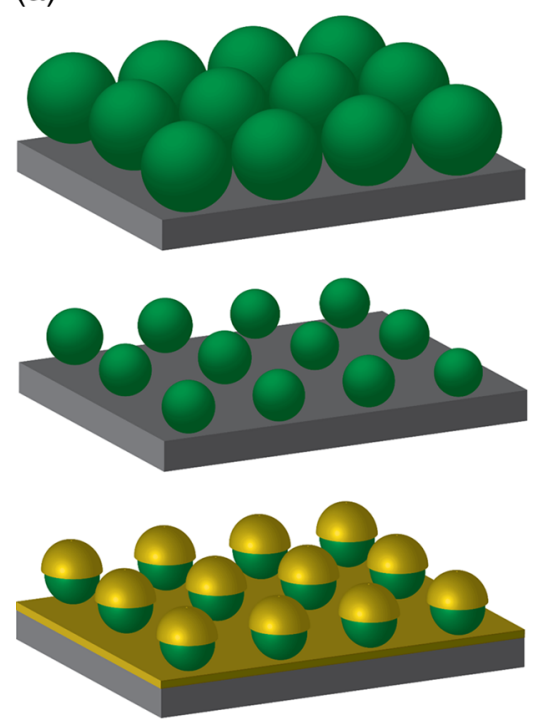

(b)
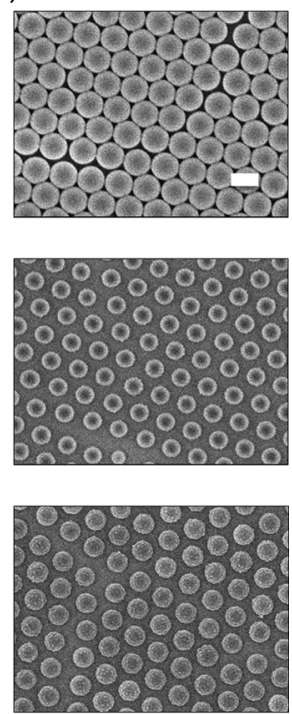

Fig. 1 (a) Schematic of the monodisperse gold nanocup fabrication technique with representative (b) scanning electron micrographs for each step. Scale bar: $400 \mathrm{~nm}$. Step 1: spin coat a monolayer of hexagonally close-packed polystyrene nanospheres on a silicon wafer. Step 2: Briefly anneal to improve adhesion to the substrate and then use an oxygen plasma to uniformly etch the nanospheres to the desired size. Step 3: Sputter a thin layer of gold $(20 \mathrm{~nm})$ at normal incidence to form nanocup structures.

(a)
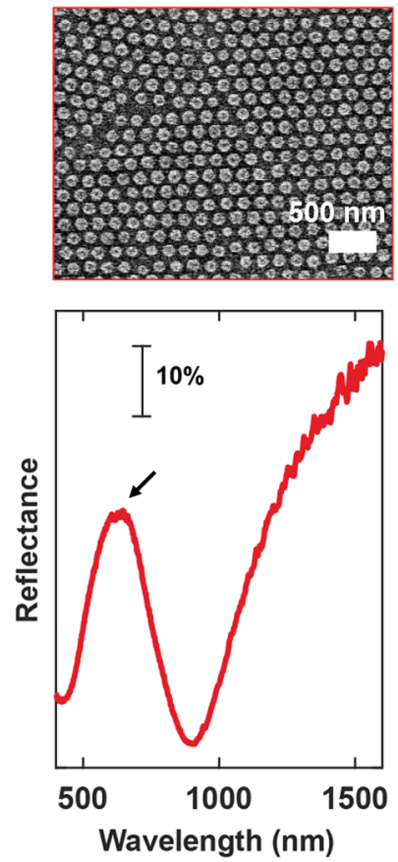

(b)
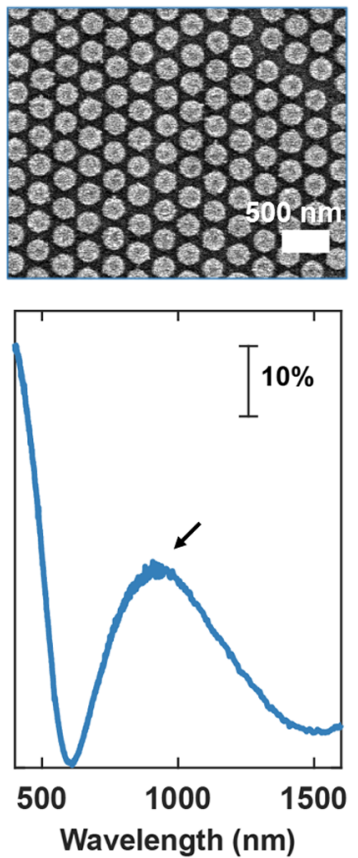

(c)
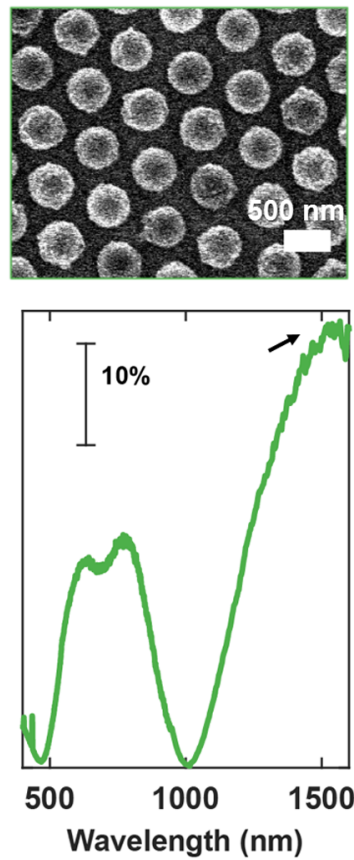

Fig. 2 Scanning electron micrographs and reflectance spectra of periodic arrays of gold nanocups bound to a gold-coated silicon substrate (before peel-off) with an average diameter and spacing of (a) 90-nm diameter/170-nm spacing, (b) 210-nm diameter/280-nm spacing, and (c) 450-nm diameter/580-nm spacing. An arrow indicates the plasmon mode of interest. Scale bar: $500 \mathrm{~nm}$. 
each sample is indicated by an arrow. Reflectance spectra were obtained by illuminating the sample at normal incidence with a fiber-coupled deuterium/halogen light source (Avalight DH-S-BAL) using a reflection probe (0.22 NA, Thorlabs RP-23) and then coupled into either a visible (AvaSpec-ULS2048) or near-IR (AvaSpec-NIR256-1.7TEC) spectrometer. The spectra were stitched at $940 \mathrm{~nm}$ giving an overall spectral range of 400 to $1600 \mathrm{~nm}$ across both detectors. Nanocup arrays with responses ranging from the visible $(650 \mathrm{~nm})$ to the near-IR were obtained (910 and $1560 \mathrm{~nm}$ ). Broadening of the LSPR was observed when the nanocup size increased as a consequence of dephasing of the plasmon resonance as the nanocup dimensions approached that of the illumination wavelength. ${ }^{21}$ The full width at half maximum (FWHM) of the fabricated nanocup arrays were $\sim 270,580$, and $700 \mathrm{~nm}$ (extrapolated because our detector cuts off at $1600 \mathrm{~nm}$ ) for the 90-, 210-, and 450-nm diameter nanocups, respectively.

Transparent and flexible nanocup films were fabricated by gently placing a $\sim 50-\mu \mathrm{m}$ polyolefin-backed film with pressure-sensitive acrylic adhesive (3M Transparent Tape 600) on top of a 2-inch metallized nanocup wafer (Fig. 3). The tape was allowed to adhere for a few minutes and then carefully removed to peel off the nanocups from the wafer. As shown by the optical photographs and confirmed by the SEM micrographs in Fig. 3, this rapid technique was highly successful at dislodging the nanocups and did not require repeated degassing steps and long curing times (in excess of $6 \mathrm{~h}$ at $40^{\circ} \mathrm{C}$ ) like certain elastomers such as poly(dimethylsiloxane) (PDMS) commonly require. ${ }^{16}$ Minor defects in the film caused by air bubbles and a few remaining nanostructures on the substrate were observed. Nonetheless, it is expected with improved process development that these imperfections can be overcome. This technique results in a similar encapsulation of the nanocups as the PDMS method, but is significantly more time efficient. Although it was not the goal of this work, we also note that the nanohole array (which did not require FIB milling) left on the substrate has numerous useful applications because it facilitates the excitation of surface plasmon polaritons.

The finite-difference time-domain (FDTD) method is a popular technique used in optical modeling of periodic nanopatterned substrates. The FDTD method works by numerically solving Maxwell's equations using finite-difference equations on a cubic mesh and Fourier transforming those time-domain results into the frequency domain. Here, we used FDTD (Synopsis FullWAVE) to simulate periodic arrays of gold nanocups ( $20 \mathrm{~nm}$ Au thickness) embedded within a poly(methyl methacrylate) (PMMA) matrix to model an analogous environment for the nanocups bound to the adhesive film (Fig. 4). PMMA's optical constants were used because they have similar optical properties to that of the polyolefin/acrylic film. For simplicity of modeling, we assumed complete encapsulation of the nanocups within the PMMA matrix. Furthermore, we only simulated single layers due to the computational complexity of simulating multilayered $50-\mu \mathrm{m}$ slabs with nanostructured features.
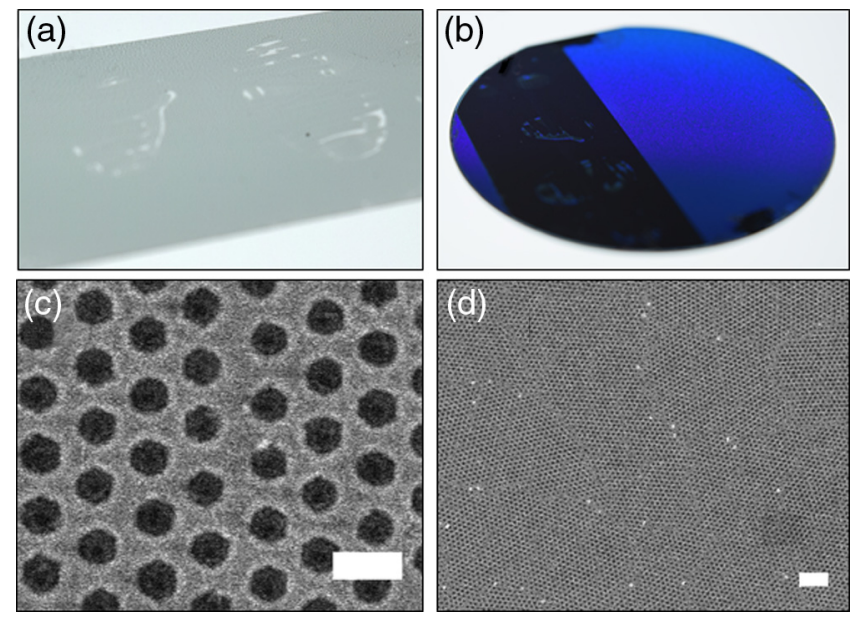

Fig. 3 (a) Representative optical image of a peeled nanocup transparent film and (b) the metallized wafer after peel-off. (c) High-magnification (scale bar: $200 \mathrm{~nm}$ ) and (d) low-magnification (scale bar: $1000 \mathrm{~nm}$ ) scanning electron micrographs of the metallized wafer after peel-off. 

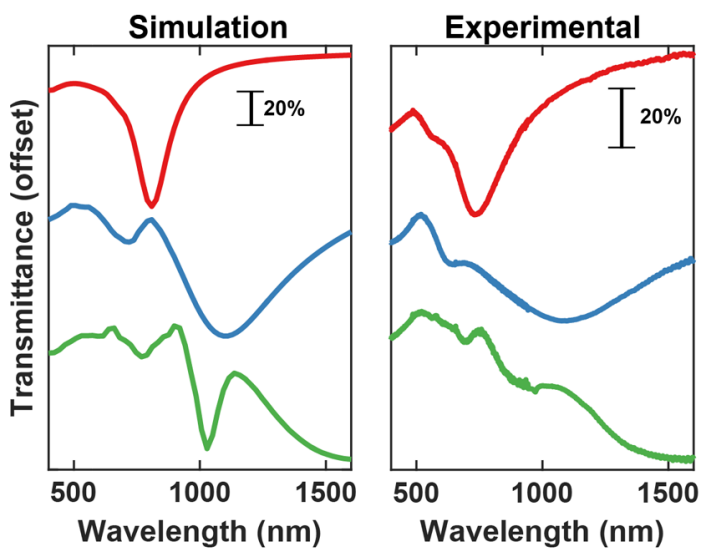

Fig. 4 Comparison of the simulated and experimental transmittance spectra of the fabricated nanoplasmonic films. In the simulated spectra, the gold nanocups were embedded within a PMMA matrix for: $90 \mathrm{~nm} / 180 \mathrm{~nm}$ (red), $210 \mathrm{~nm} / 310 \mathrm{~nm}$ (blue), and $450 \mathrm{~nm} / 580 \mathrm{~nm}$ (green) diameter/spacing structures. The experimental spectra correspond to representative spectra of gold nanocups with average diameters of $90 \mathrm{~nm}$ (red), $210 \mathrm{~nm}$ (blue), and $450 \mathrm{~nm}$ (green).

After peel-off, the transmittance spectra of the nanocup films were measured individually and for multiple configurations of two layer stacks (Fig. 5). Transmittance spectra were collected in an analogous manner to the reflectance spectra, with the exception that illumination took place $180 \mathrm{deg}$ with respect to the collection probe. The spectra were postprocessed with a moving average filter to remove etalon fringes caused by the $50-\mu \mathrm{m}$ thick adhesive tape. In the individual films, we observed red shifting of the LSPR (e.g., 650 to $730 \mathrm{~nm}$ and 910 to $1100 \mathrm{~nm}$ ) as a consequence of the nanocups being embedded within a new dielectric environment.

The single layer experimental and simulated data match well in terms of both resonance frequency and qualitative spectral characteristics. The plasmon resonances of interest for the simulated single layer nanofilms (Fig. 4) are at 810, 1090, and $1630 \mathrm{~nm}$ for the 90-, 210-, and 450-nm nanocup arrays, respectively. The FWHM of the simulated films at these resonances are 200, 500, and $900 \mathrm{~nm}$. These dominant resonances are the primary contributors to the spectral characteristics of the stacked films that are created when layering films; nonetheless,

(a)
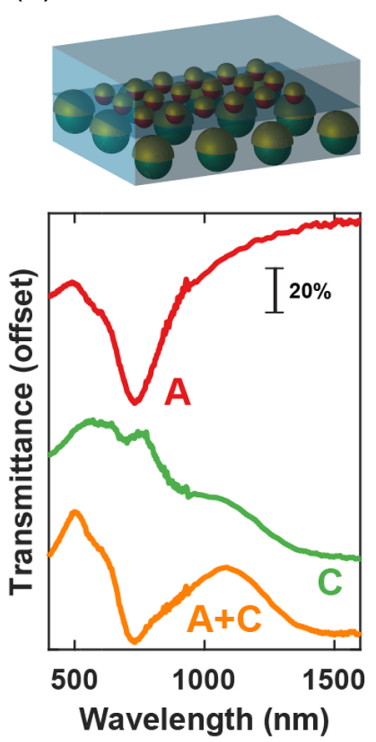

(b)
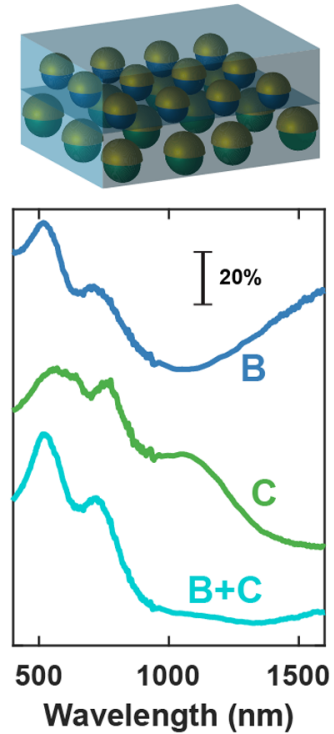

(c)
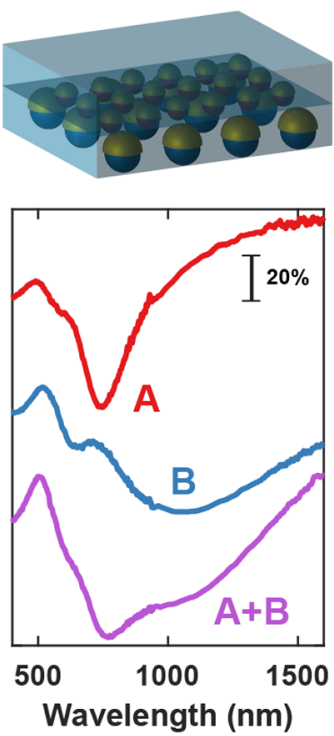

Fig. 5 Transmittance spectra of individual films (A: red, B: blue, and C: green) and two layer stacked films for (a) 90-nm and 450-nm nanocups ( $+C$, orange), (b) 210-nm and 450-nm nanocups $(B+C$, cyan), and (c) $90-n m$ and 210-nm nanocups $(A+B$, purple). 
lower energy resonances ${ }^{18}$ are observable, especially in the cases of the 210- and 450-nm arrays. Compared with the experimental data of single films (Fig. 5), the simulated resonances are generally narrower, as is expected because experimental substrates have a degree of variance in polymeric nanosphere size, metal thickness, and additional defects. The 450-nm diameter gold nanocup simulation contains a sharp band just past $1000 \mathrm{~nm}$ that is not present in the experimental data. This may be an optical interference effect arising from the array of polystyrene nanosphere cores. Further investigation into the theoretical properties of these films will be necessary to fully identify its origins. The experimental data likely does not exhibit this band because of defects in the metal deposition film as well as point defects and grain boundaries in the self-assembled film leading to a reduction in quality and, therefore, broadening of the measured spectra.

The plasmon resonances broadened after peel-off, especially for the samples with a near-IR response. A unique multiresonant spectral response was obtained when the films were layered (orange, cyan, and purple traces). In Fig. 5(a), the plasmon resonances were spaced considerably and two distinct resonances were observed near 730 and $1500 \mathrm{~nm}$. In the other two cases [Figs. 5(b) and 5(c)], the plasmon resonances of the individual films were closer together and the subsequent measurements of the stacked film resulted in a unique but broad response.

To further extend the idea of multiresonant films, we fabricated a three layer stack (Fig. 6). The additive combination of the three plasmon resonances of the individual constituent films can clearly be seen, although they are somewhat muted with the strongest resonance being blue shifted to $660 \mathrm{~nm}$. Because the nanofilms are not completely transparent in the visible ( $\sim 66 \%$ average transmission per film), the three layer stack only transmits $\sim 20 \%$ light from 400 to $750 \mathrm{~nm}$, whereas the two layer stack transmitted $\sim 40 \%$ light. The loss of transmission of light likely arises from thin film interference effects from the metal-coated polystyrene spheres, gold's interband transitions ${ }^{22}$ in the blue $(\sim 470 \mathrm{~nm})$, and scattering losses from the nanocups. Because the tape is highly transparent throughout the visible and near-IR, we did not observe any degradation of the plasmonic response due to stacking multiple $50-\mu \mathrm{m}$ films. The attenuation of the transmission signal almost exclusively occurred as a result of the nanocups rather than the relatively thick polyolefin films.

In this work, we demonstrated multiresonant plasmonic substrates capable of spanning a wide portion of the visible and near-IR spectrum. Transfer of gold nanocups to pressure-sensitive adhesive film is a rapid and easy method for preparing flexible, optically transparent films. Our experimental results match the simulated results of single layer films, which confirm the nanoplasmonic optical mechanism. As expected, shifting of the plasmon resonance is observed when altering the nanocup's local dielectric environment by way of encapsulating it into an acrylic adhesive. Stacking of the nanofilms resulted in unique spectral responses that would traditionally require serial processes, such as EBL to attain.

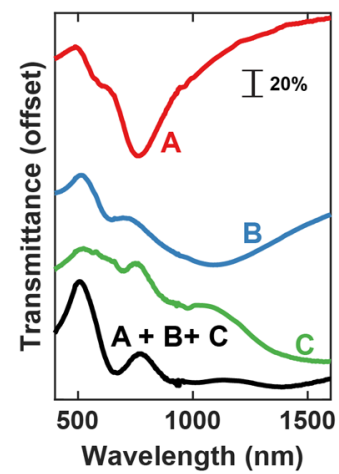

(a)

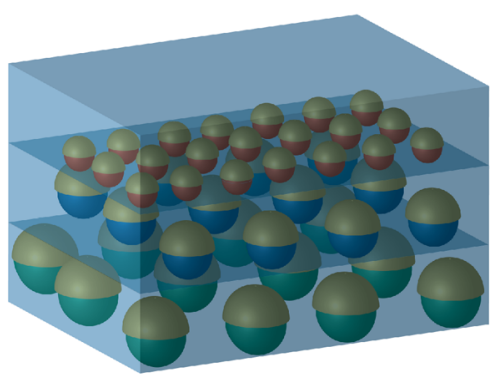

(b)

Fig. 6 (a) Transmittance spectra of three individual gold nanocup films transferred (A: red, B: blue, and $C$ : green spectra) and stacked in three layers $(A+B+C)$. The plasmon resonance of interest for each individual film is indicated by an arrow. The resulting spectra after stacking are shown in black. (b) Cartoon schematic of the three layer stacked film, where complete encapsulation of the nanostructures by the pressure-sensitive adhesive film is assumed. 


\section{Acknowledgments}

This research was performed at the Pacific Northwest National Laboratory (PNNL), which is operated by Battelle Memorial Institute for the Department of Energy (DOE) under Contract No. DE-AC05-76RL01830. The authors gratefully acknowledge support from the U.S. Department of State through the Key Verification Assets Fund (V Fund) under Interagency Agreement SIAA15AVCVPO10.

\section{References}

1. N. S. King et al., "Fano resonant aluminum nanoclusters for plasmonic colorimetric sensing," ACS Nano 9(11), 10628-10636 (2015).

2. J. C. Ndukaife, V. M. Shalaev, and A. Boltasseva, "Plasmonics-turning loss into gain," Science 351(6271), 334-335 (2016).

3. A. B. Taylor et al., "Electron-beam lithography of plasmonic nanorod arrays for multilayered optical storage," Opt. Express 22(11), 13234-13243 (2014).

4. Q. Gan, F. J. Bartoli, and Z. H. Kafafi, "Plasmonic-enhanced organic photovoltaics: breaking the $10 \%$ efficiency barrier," Adv. Mater. 25(17), 2385-2396 (2013).

5. J.-F. Li et al., "Dielectric shell isolated and graphene shell isolated nanoparticle enhanced Raman spectroscopies and their applications," Chem. Soc. Rev. 44(23), 8399-8409 (2015).

6. K. M. McPeak et al., "Plasmonic films can easily be better: rules and recipes," ACS Photonics 2(3), 326-333 (2015).

7. P. R. Sajanlal et al., "Anisotropic nanomaterials: structure, growth, assembly, and functions," Nano Rev. 2, 5883 (2011).

8. T. Paik et al., "Binary and ternary superlattices self-assembled from colloidal nanodisks and nanorods," J. Am. Chem. Soc. 137(20), 6662-6669 (2015).

9. P. Wang et al., "Programming self-assembly of DNA origami honeycomb two-dimensional lattices and plasmonic metamaterials," J. Am. Chem. Soc. 138(24), 7733-7740 (2016).

10. K. Thorkelsson, P. Bai, and T. Xu, "Self-assembly and applications of anisotropic nanomaterials: a review," Nano Today 10(1), 48-66 (2015).

11. S. E. Lohse et al., "A simple millifluidic benchtop reactor system for the high-throughput synthesis and functionalization of gold nanoparticles with different sizes and shapes," ACS Nano 7(5), 4135-4150 (2013).

12. L. V. Brown et al., "Fan-shaped gold nanoantennas above reflective substrates for surfaceenhanced infrared absorption (SEIRA)," Nano Lett. 15(2), 1272-1280 (2015).

13. C.-Y. Wang et al., "Giant colloidal silver crystals for low-loss linear and nonlinear plasmonics," Nat. Commun. 6, 7734 (2015).

14. B. Ai et al., "Advanced colloidal lithography beyond surface patterning," Adv. Mater. Interfaces 4(1) (2016).

15. N. S. King et al., "Angle- and spectral-dependent light scattering from plasmonic nanocups," ACS Nano 5(9), 7254-7262 (2011).

16. N. A. Mirin and N. J. Halas, "Light-bending nanoparticles," Nano Lett. 9(3), 1255-1259 (2009).

17. Y. Zhang et al., "Orientation-preserving transfer and directional light scattering from individual light-bending nanoparticles," Nano Lett. 11(4), 1838-1844 (2011).

18. L. Wollet et al., "Plasmon hybridization in stacked metallic nanocups," Opt. Mater. Express 2(10), 1384-1390 (2012).

19. C. J. Choi and S. Semancik, "Multi-resonant plasmonic nanodome arrays for label-free biosensing applications," Nanoscale 5(17), 8138-8145 (2013).

20. K. Chen et al., "Moiré nanosphere lithography," ACS Nano 9(6), 6031-6040 (2015).

21. P. Mulvaney, "Not all that's gold does glitter," MRS Bull. 26(12), 1009-1014 (2001).

22. P. G. Etchegoin, E. C. Le Ru, and M. Meyer, "An analytic model for the optical properties of gold," J. Chem. Phys. 125(16), 164705 (2006).

Brent M. DeVetter received his $\mathrm{PhD}$ from the University of Illinois at Urbana-Champaign in 2016. He is currently a postdoctoral research associate at the Pacific Northwest National 
Laboratory in Richland, Washington. His research interests include plasmonics, vibrational spectroscopy, and materials synthesis.

Bruce E. Bernacki received his $\mathrm{PhD}$ in optical sciences from the University of Arizona in 1992 and has been a senior research scientist at PNNL since 2005. His research interests include optical design and modeling, infrared laser absorption spectroscopy, infrared imaging spectroscopy, optical instrumentation and passive millimeter wave sensing.

Wendy D. Bennett is currently a senior research scientist at the Pacific Northwest National Laboratory in Richland, Washington. She has 39 years experience with modeling, PVD deposition and characterization of thin film coatings. She holds patents in 3 technology areas: thin film coatings, microtechnology, and lithium thin film batteries.

Alan Schemer-Kohrn is currently a senior laboratory technician with over 20 years of experience in electron microscopy, 10 years of experience as a field service representative for an electron microscope vendor, and 10 years at PNNL concentrating on high resolution imaging and elemental analysis of samples with the SEM.

Kyle J. Alvine received his $\mathrm{PhD}$ in applied physics from Harvard University in 2006 and then completed a NIST-NRC postdoctoral fellowship at NIST, Gaithersburg, Maryland. Since 2008, he has been a senior research scientist at the Pacific Northwest National Laboratory. His research interests include metamaterials, nanostructured optics, nanofabrication, surface science, and self-assembly. 